\title{
Application of network pharmacology and molecular docking to elucidate the potential mechanism of Eucommia ulmoides-Radix Achyranthis Bidentatae against osteoarthritis
}

\author{
Gong-hui Jian, Bing-zhu Su, Wen-jia Zhou and Hui Xiong ${ }^{*}$ (i)
}

\author{
*Correspondence: xh_hn@hnucm. \\ edu.cn \\ Hunan University of Chinese \\ Medicine, Changsha, Hunan \\ Province, People's Republic of China
}

\begin{abstract}
Background: Osteoarthritis is a disabling disease, which seriously affects the quality of life of patients. Increasing evidence has indicated that Chinese herbal medicine including Eucommia ulmoides (EU) and Radix Achyranthis Bidentatae (RAB) have potential in the treatment of osteoarthritis, and this is associated with their multitarget and multi-link action characteristics. Although their potential anti-arthritic activity has been reported, the exact mechanism of EU-RAB action in osteoarthritis remains unexplored. Therefore, this study explores the mechanism of EU-RAB against osteoarthritis using network pharmacology and molecular docking technology.

Methods: Public databases including TCMSP、BATMAN-TCM、OMIM and Genecards were used to predict the bioactive ingredients and putative targets of EU-RAB against osteoarthritis. Enrichment analysis was performed to expound the biological functions and associated pathways of the hub targets. Cytoscape software was used to construct a "compounds-targets-pathways" network for elucidating the comprehensive molecular mechanism of EU-RAB against osteoarthritis. Molecular docking was used to verify the correlation between the main active ingredients and hub targets.

Results: Network pharmacological analysis of EU-RAB in the treatment of osteoarthritis, identified 50 active ingredients including quercetin, kaempferol, wogonin, and baicalein with important biological effect. A total of 68 key targets were screened, including IL-6, EGFR, MAPK8, etc., and they were found to be enriched in a series of signaling pathways, such as apoptosis, TNF, MAPK, PI3K/AKT, and IL-17 signaling pathways. Moreover, molecular docking analysis showed that the main ingredients were tightly bound to the core targets, further confirming the anti-arthritic effects.

(Continued on next page)
\end{abstract}

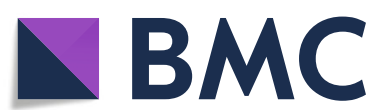

C The Author(s) 2020 Open Access This article is licensed under a Creative Commons Attribution 4.0 International License, which permits use, sharing, adaptation, distribution and reproduction in any medium or format, as long as you give appropriate credit to the original author(s) and the source, provide a link to the Creative Commons licence, and indicate if changes were made. The images or other third party material in this article are included in the article's Creative Commons licence, unless indicated otherwise in a credit line to the material. If material is not included in the article's Creative Commons licence and your intended use is not permitted by statutory regulation or exceeds the permitted use, you will need to obtain permission directly from the copyright holder. To view a copy of this licence, visit http://creativecommons.org/licenses/by/4.0/. The Creative Commons Public Domain Dedication waiver (http://creativecommons.org/publicdomain/zero/1.0/) applies to the data made available in this article, unless otherwise stated in a credit line to the data. 
(Continued from previous page)

Conclusion: Based on network pharmacology and molecular docking analysis, the present study provides insights into the potential mechanism of EU-RAB in osteoarthritis after successfully screening for associated key target genes and signaling pathways. These findings further provide a theoretical basis for further pharmacological research into the potential mechanism of EU-RAB in osteoarthritis.

Keywords: Osteoarthritis, Network pharmacology, Traditional Chinese medicine, Eucommia ulmoides, Radix Achyranthis Bidentatae, Molecular docking

\section{Introduction}

Osteoarthritis $(\mathrm{OA})$ is characterized by progressive degeneration and wear of cartilage as well as subchondral osteosclerosis, pain, swelling, and stiffness in the joints. Globally, osteoarthritis affects approximately 250 million people [1]. Chronic pain and disability associated with OA can lead to anxiety, depression, and suicidal emotion [2]. However, long-term use of non-steroidal anti-inflammatory drugs in patients with OA is associated with gastrointestinal and cardiovascular side-effects. Recent therapies in regenerative medicine including stem cell and platelet-rich plasma therapy are commonly used in the short-term treatment of localized pains such as joint pain [3]. Therefore, there is an urgent need for systematic and effective clinical treatment approaches to reduce chronic pain and improve the quality of life in patients with OA.

In the field of Traditional Chinese Medicine (TCM), OA is referred to as Arthralgia Syndrome, which originated from the ancient book "Huangdi Neijing". Valuable research over the years has explored the role of TCM in the treatment of OA, via the mechanisms of anti-inflammation, anti-apoptosis, and anti-catabolism [4]. Eucommia ulmoides (EU, Chinese name: Duzhong) and Radix Achyranthis Bidentatae (RAB, Chinese name: Niuxi), are natural herbs reported to nourish kidney and strengthen the bone and are recorded in the classic of Herbal medicine Shennong Ben Cao Jing, which is one of China's earliest pharmaceutical works $[5,6]$. Research shows that the EU is currently the most widely used traditional Chinese medicine in the treatment of OA, while RAB is ranked third [7]. Moreover, both are often used and recognized as classical pair drugs playing a synergistic role in anti-osteoarthritis $[8,9]$. Xie et al. showed that EU extract suppresses the activity of the PI3K/Akt pathway to mitigate the release of inflammatory factors which play a significant role in anti-osteoarthritis [10]. Zhang et al. demonstrated that EU extract inhibited bone loss and maintained metabolic balance [11]. Ecdysterone and saponins extracted from RAB were reported to protect chondrocytes through anti-apoptosis and anti-inflammation effects [12, 13]. Weng et al. revealed the positive effect of Achyranthes bidentata polysaccharides on chondrocytes proliferation [14]. Therefore, it is expected that a mixture of EU and RAB is capable of having potent anti-osteoarthritis effects. However, the potential pharmacological mechanisms of action of EU-RAB and their interaction with osteoarthritis-related targets and pathways remain unknown and need to be further explored.

TCM has a complex mechanism of action and multi-pathway interactions. Over the past few decades, Chinese medicine was regarded as empirical medicine, which was neglected due to a lack of modernized research approaches in related disciplines such as molecular biology, pharmacology, and bioinformatics, etc. [15]. Network pharmacology 
is a novel method that combines computer science with medicine. Based on" multi-gene, multi-target, multi-pathway" interaction network, network pharmacology combined with TCM provides an in-depth understanding of the active ingredients in Chinese herbs [16]. Besides, this also provides potent evidence into biological targets and the underlying mechanism of action of TCM in the treatment of various diseases [17]. This study seeks to elucidate the molecular targets and underlying mechanism of action of EU-RAB in osteoarthritis using network pharmacology and molecular docking (Fig. 1).

\section{Methods}

\section{Identification of active ingredients in EU-RAB}

The compounds-related data of EU and RAB were retrieved from Traditional Chinese Medicine Systems Pharmacology (TCMSP) database (http://lsp.nwu.edu.cn/tcmsp.php), which is a unique platform for the analysis of active ingredients of Chinese herbal medicine and their interaction in specific diseases [18], and the Bioinformatics Analysis Tool for Molecular mechanism of Traditional Chinese Medicine (BATMAN-TCM) database (http://bionet. ncpsb.org/batman-tcm/), which is the first online bioinformatics analysis tool for screening

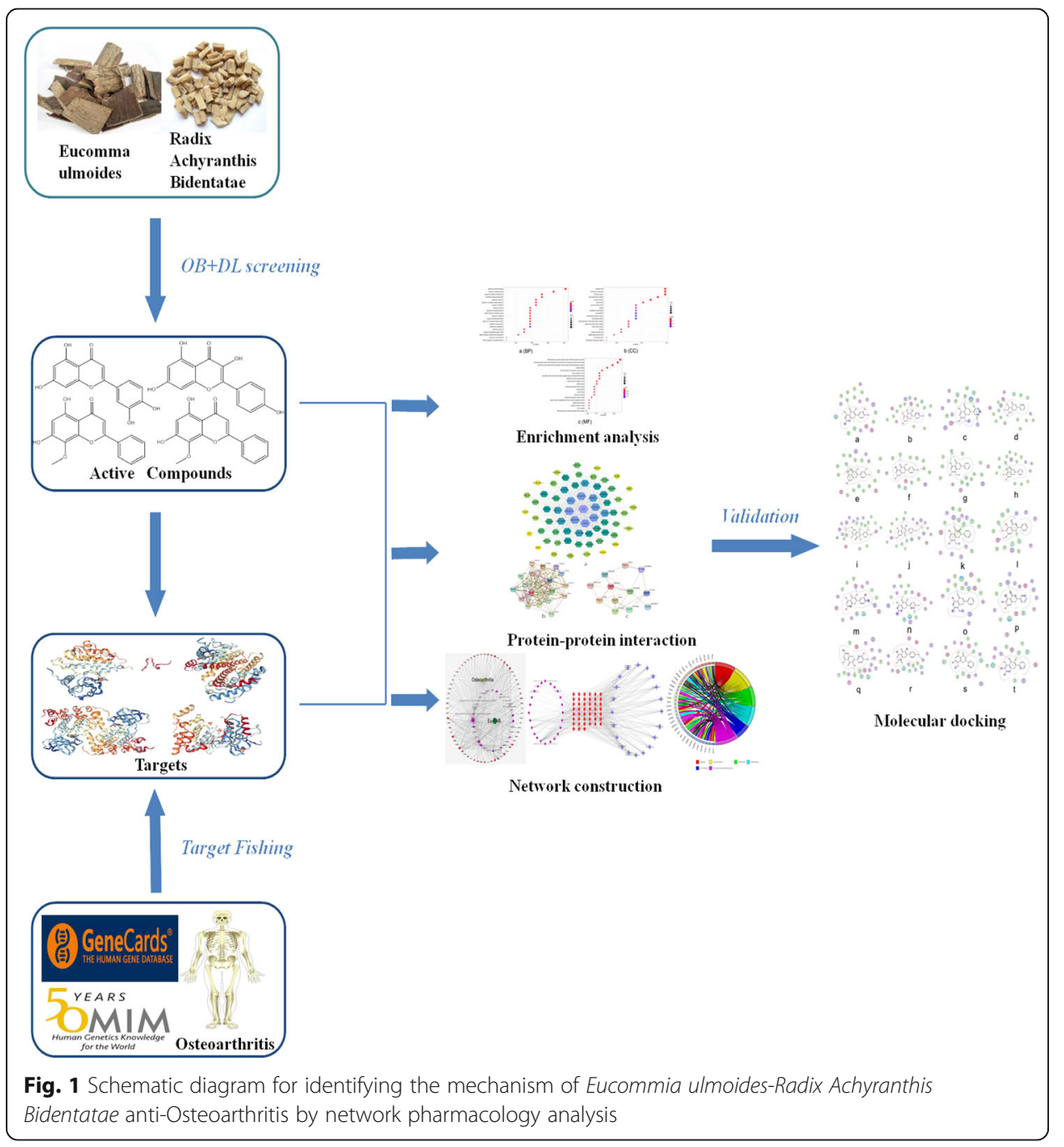


of the molecular mechanism of TCM [19]. Oral bioavailability (OB) and drug-likeness (DL) are important pharmacokinetic parameters used to identify and screen active ingredients based on the ADME (absorption, distribution, metabolism, and excretion) model [20].

$\mathrm{OB}$ represents the relative amount and rate at which the drug is orally absorbed into the body circulation. Most TCM compounds are difficult to reach specific tissues and exert certain pharmacological effects, mainly due to poor OB [21]. In the TCMSP database, a widespread computer prediction model OBioavail 1.1 [22] has been used to calculate the $\mathrm{OB}$ value of compounds. Based on the recommendation in the TCMSP database and previous studies [23], compounds with $\mathrm{OB} \geq 30 \%$ are considered to have reliable pharmacological activity.

DL is a concept that is based on the physical and chemical properties and molecular structure of existing drugs and is used to evaluate whether a compound meets the conditions to become a new drug. In this study, the Tanimoto coefficient [24] was used to calculate the DL (see Eq. (1)) of each compound in EU-RAB.

$$
\mathrm{F}(\mathrm{X}, \mathrm{Y})=\frac{X Y}{(|X| 2+|Y| 2-X Y)}
$$

In this equation, $\mathrm{X}$ represents the molecular descriptors of a new ingredient, and $\mathrm{Y}$ represents the average molecular properties of 6511 molecules in the DrugBank database (http://www.drugbank.ca). The DL threshold is determined based on the average DL value of the Drugbank being 0.18.

In the database, we obtained the corresponding ingredients by inputting the names of the herbal medicine and then selecting the active ingredients for further analysis using the criteria: $\mathrm{OB} \geq 30 \%$ and $\mathrm{DL} \geq 0.18$.

\section{The putative targets of EU-RAB}

TCMSP database not only allows users to analyze to identify active compounds of Chinese herbal medicine but to also search for related genes targets of the active compounds. After obtaining the active ingredients by limiting $\mathrm{OB}$ and DL, the targets of each ingredient were collected and the repeating parts were removed to obtain the EURAB related targets. The species of target proteins were set to "Homo sapiens" and the whole gene information including gene name, gene ID, gene symbol was obtained using Uniprot protein sequence resource (https://www.uniprot.org).

\section{Related targets of osteoarthritis and prediction of potential targets of EU-RAB against osteoarthritis}

Osteoarthritis targets were acquired from two databases. GeneCards (https://www.genecards.org/, ver. 4.9.0) database is a human gene online analysis platform which is based on abundant gene resources, it integrates and predicts gene targets associated with diseases and also used to conduct Gene-Centered "OMIC" research [25]. OMIM (http:// www.omim.org/, updated February 19, 2019) database is centered on the connection between the gene and phenotype, maintained timely updating [26]. The keyword "osteoarthritis" was searched in the two databases to obtain the disease-targets; then, the overlapping part after integrating the disease-targets and drug-targets were the potential targets of EU-RAB against osteoarthritis. 


\section{Protein-protein interaction}

Protein-protein interaction (PPI) analysis helped in the identification of key targets belonging to both EU-RAB and OA, and also highlight the related hub genes of EU-RAB anti-OA. The active ingredient of EU-RAB relevant to OA targets was retrieved via the STRING (https://string-db.org/,ver. 11.0) database, mined the key regulatory genes. The potential targets were inputted to "Multiple proteins" in STRING and the organism was limited to "Homo sapiens". A confidence score with correlation degree $\geq 0.400$ was set using the default threshold, and this enabled the enhanced interaction between targets while ensuring a positive rate and extracted the PPI network after removing the discrete genes.

In the PPI network, many closely interconnected regions were defined as clusters or topology modules, which were constructed by each node and edge as well as made to reflect on related molecular biological functions and core protein processes [27]. Clusters can be obtained using MCODE, a plug-in of Cytoscape 3.7.2. Each cluster of proteins was inputted, to reproduce the corresponding cluster map and predict functional links between proteins.

\section{Enrichment analysis}

Gene ontology (GO) and Kyoto Encyclopedia of Genes and Genomes (KEGG) pathway enrichment analyses were conducted to explore the core mechanism of action and biological pathways associated with the drug. After the PPI network was successfully constructed, hub co-targets were imported into $\mathrm{R}$ software and the clusterprofile, a bioconductor package, was used to perform the GO and KEGG enrichment analysis, to obtain the biological processes, cellular components, molecular function and key signaling pathways. Only functional annotations with enrichment $p$ values smaller than 0.05 were chosen for further analysis.

\section{Network construction}

Network construction was performed using Cytoscape (ver. 3.7.2), a network visualization software to intuitively display the compound-target-pathway relationship between EU-RAB and OA, including 3 networks, namely: 1) compound-target network of EU-RAB; 2) potential compounds-potential targets network of EU-RAB for antiosteoarthritis; 3) potential compounds-potential targets-pathways network of EU-RAB for anti-osteoarthritis.

\section{Verification of compound-target interaction through molecular docking}

Molecular docking technology was based on the "Key-Lock principle" to reflect the small molecule ligands-protein receptors association. Further, the best binding mode and site were searched based on the conformation change and energy matching process. In this study, the Molecular Operating Environment (MOE) (v2015.10) was performed to validate compound-protein target interaction. The crystal structure of the protein targets was downloaded from the RCSB PDB database (http://www.rcsb.org/). The imported crystal structure was imported into MOE for use in constructing the receptor protein grid, which was associated with the processes of protonation, removal of water molecules and repetitive structure, structure preparation, and energy minimization. The receptor grid construction was used to select the binding site with ligands. Lastly, the three-dimensional 
compound structure was edited in ChemBioDraw software, imported into MOE, and docked with protein construct to obtain the graphical results of molecular docking.

\section{Results}

Active compounds of EU-RAB

A total of 433 EU-RAB compounds were retrieved from the TCMSP and BATMANTCM database. Based on the screening criteria, $\mathrm{OB} \geq 30 \%$ and $\mathrm{DL} \geq 0.18,31$ active ingredients in the EU, and 22 active ingredients in RAB were obtained. There were 50 active ingredients after deleting repetitions, such as quercetin, kaempferol, and wogonin. The details are shown in the Supplementary file, Table S1.

\section{Putative targets of EU-RAB}

In this study, 102 putative targets for the $\mathrm{EU}$, and 100 putative targets for RAB were identified. However, most of the targets were repetitive, revealing that the active ingredients of the EU and RAB may possess identical biological effects and achieve a cooperative effect when combined. A total of 110 putative targets of EU-RAB (Supplementary file, Table S2) were obtained by further integrating all the targets.

\section{Gene targets of $O A$ and potential targets of EU-RAB against $O A$}

A total of 2498 OA related gene targets were retrieved from the OMIM and Genecards databases. Further, 68 putative targets of EU-RAB were obtained after integrating and connecting 110 putative targets of EU-RAB with 2498 OA gene targets. IL-6, EGFR, GSK3 $\beta$, ESR, etc. are some of the densely related targets identified between EU-RAB and $\mathrm{OA}$, and details are shown in Table 1.

\section{Enrichment analysis}

GO and KEGG pathway enrichment analysis contributed to further analysis of cellular localization, biological effect, pathway, and function of the 68 gene targets of EU-RAB anti-OA. In GO analysis, the 68 potential genes were highly enriched in 1272 biological processes (BP), 31 cellular components (CC), and 95 molecular functions (MF) with $p$ value $<0.05$. As shown in Fig. 2 , response to steroid hormone $\left(p=3.91 \mathrm{E}^{-17}, \mathrm{FDR}=\right.$ $\left.1.99 \mathrm{E}^{-17}\right)$, regulation of apoptotic signaling pathway $\left(p=1.16 \mathrm{E}^{-11}, \mathrm{FDR}=5.88 \mathrm{E}^{-12}\right)$, response to oxidative stress $\left(p=6.98 \mathrm{E}^{-14}, \mathrm{FDR}=3.55 \mathrm{E}^{-14}\right)$ and cellular response to oxidative stress $\left(p=3.75 \mathrm{E}^{-12}, \mathrm{FDR}=1.91 \mathrm{E}^{-12}\right)$ were the closely related to $\mathrm{OA}$ biological processes. In regard to cellular components, higher enrichment was found in membrane raft $\left(p=9.25 \mathrm{E}^{-06}, \mathrm{FDR}=6.84 \mathrm{E}^{-06}\right)$, membrane microdomain $\left(p=9.25 \mathrm{E}^{-06}\right.$, $\left.\mathrm{FDR}=6.84 \mathrm{E}^{-06}\right)$ and membrane region $\left(p=9.25 \mathrm{E}^{-06}, \mathrm{FDR}=6.84 \mathrm{E}^{-06}\right)$. The main OArelated terms in molecular functions contained DNA-binding transcription activator activity, RNA polymerase II-specific $\left(p=1.56 \mathrm{E}^{-09}, \mathrm{FDR}=8.48 \mathrm{E}^{-10}\right)$ and cysteine-type endopeptidase activity involved in apoptotic process $\left(p=9.18 \mathrm{E}^{-06}, \mathrm{FDR}=5.00 \mathrm{E}^{-06}\right)$ and estrogen receptor binding $\left(p=3.11 \mathrm{E}^{-04}, \mathrm{FDR}=1.70 \mathrm{E}^{-04}\right)$.

KEGG enrichment analysis focused on the anti-osteoarthrits pathways of EU-RAB. Results demonstrated that "Apoptosis", "TNF signaling pathway", "PI3K-Akt signaling pathway", "MAPK signaling pathway" and "IL-17 signaling pathway" were highly associated with the onset and progression of osteoarthritis (Table 2 and Fig. 3). Besides, 
Table 1 Potential targets of Eucommia ulmoides (EU)-Radix Achyranthis Bidentatae (RAB) against Osteoarthritis (OA)

\begin{tabular}{llllllll}
\hline N0. & Gene & No & Gene & N0 & Gene & N0 & Gene \\
\hline 1 & PTGS1 & 18 & NFKBIA & 35 & CCNB1 & 52 & GSTM1 \\
2 & AR & 19 & POR & 36 & ALOX5 & 53 & PGR \\
3 & PPARG & 20 & CASP8 & 37 & GSTP1 & 54 & ESR1 \\
4 & F7 & 21 & PRKCA & 38 & NFE2L2 & 55 & IKBKB \\
5 & RELA & 22 & HIF1A & 39 & PARP1 & 56 & MAPK8 \\
6 & EGFR & 23 & ERBB2 & 40 & AHR & 57 & ALB \\
7 & VEGFA & 24 & CYP3A4 & 41 & COL3A1 & 58 & CTNNB1 \\
8 & CCND1 & 25 & CAV1 & 42 & NR113 & 59 & CASP7 \\
9 & BCL2 & 26 & MYC & 43 & HSF1 & 60 & GSK3B \\
10 & FOS & 27 & CYP1A1 & 44 & CRP & 61 & DRD2 \\
11 & EIF6 & 28 & ICAM1 & 45 & RUNX2 & 62 & NR3C1 \\
12 & CASP9 & 29 & SELE & 46 & CTSD & 63 & ESR2 \\
13 & PLAU & 30 & VCAM1 & 47 & IGFBP3 & 64 & FOSL1 \\
14 & IL6 & 31 & PTGER3 & 48 & IGF2 & 65 & CYCS \\
15 & CASP3 & 32 & BIRC5 & 49 & IRF1 & 66 & NOX5 \\
16 & TP63 & 33 & NOS3 & 50 & PON1 & 67 & TEP1 \\
17 & ELK1 & 34 & HSPB1 & 51 & DIO1 & 68 & MCL1 \\
\hline & & & & & \\
\hline
\end{tabular}

"Apoptosis" was the pathway found to be the closest and with a high enrichment degree in $\mathrm{OA}$, and mapped 15 gene targets.

\section{Network construction analysis}

\section{Potential targets of EU-RAB network analysis}

The network construction of active compound-potential targets of EU-RAB using 50 compounds and 110 targets was based on the "one to multiple", "multiple to one" links between 150 nodes and 454 edges in this complex network (Fig. 4). The more edges and nodes obtained, the stronger the node interaction, and versa vice. In this network, quercetin $($ degree $=154)$, kaempferol (degree $=68)$, wogonin $($ degree $=20)$, and baicalein (degree $=19)$ were the main active ingredients of EU-RAB for the treatment of various diseases.

\section{Construction of a compound-target network of EU-RAB anti-OA}

A total of 50 active compounds and 68 hub targets were used to establish an interactive network of EU-RAB in the treatment of OA. As shown in Fig. 5, the network contained 103 nodes and 379 edges. Some compounds nodes were found to possess more edges, including quercetin $($ degree $=107)$, kaempferol $($ degree $=45)$, wogonin $($ degree $=17)$, baicalein $($ degree $=16)$, beta-sitosterol $($ degree $=17)$, etc., revealing that these compounds linked more targets and served a critical role in EU-RAB anti-OA.

\section{Construction of a compound-target-pathway network of EU-RAB anti-OA}

To further reveal the mechanism action of EU-RAB on OA, a systematic and comprehensive bioinformatics network was constructed using 50 active compounds, 68 


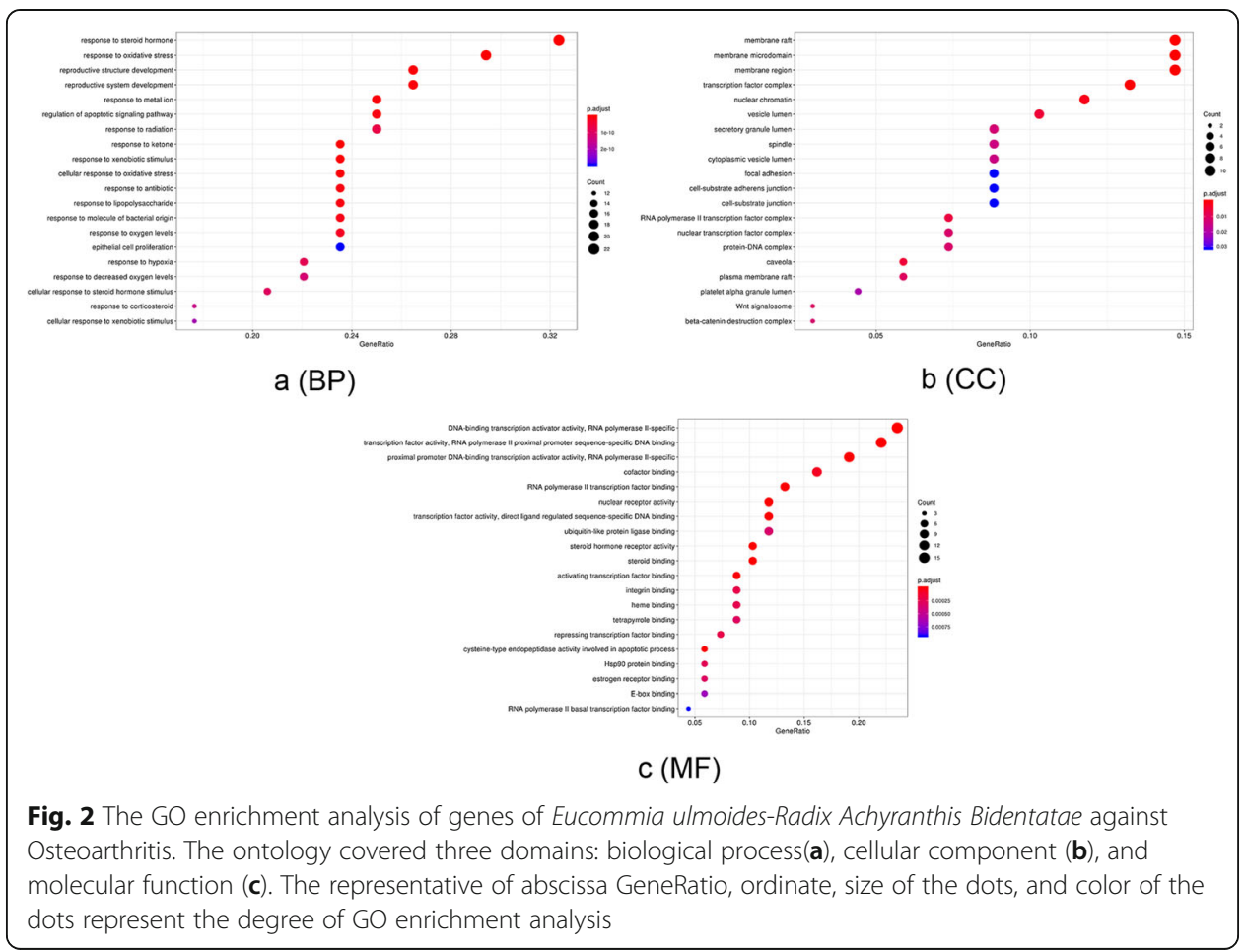

potential gene targets, and 20 KEGG signaling pathways. The complex network contained 89 nodes and 380 edges, indicating that there were complex interactions among multiple compounds-targets-pathways, which also played a synergistic effect on inhibiting the progression of OA. Some targets/pathways were also likely to play critical roles in osteoarthritis. In the bioinformatics network, associated signaling pathways including apoptosis $($ degree $=15)$, PI3K/Akt signaling pathway (degree $=15)$, Cysteinyl aspartatespecific proteinase 3 (Caspase3, degree $=21$ ), inhibitor of nuclear factor kappa-B kinase subunit beta $(\mathrm{IKBKB}$, degree $=16)$ and Glycogen Synthase Kinase 3 beta (GSK3 $\beta$, degree $=16)$ were identified. The details of the compound-target-pathway network are shown in Table 2 and Figs. 3 and 6.

\section{PPI network construction}

A total of 68 hub gene targets were imported into STRING for the construction of a protein-protein interaction network. A PPI network containing 68 nodes and 699 edges (Fig. 7a) was obtained. The core gene targets possessed higher degree and were more likely to play a key role in EU-RAB anti-osteoarthritis including, Interleukin-6 (IL-6), Vascular endothelial growth factor (VEGFA), Estrogen receptor (ESR1), Epidermal growth factor receptor (EGFR), Mitogen-activated protein kinase 8 (MAPK8) and Caspase3.

MCODE analysis of PPI network based on the STRING database and Cytoscape software resulted in 2 Clusters networks (Fig. 7b, c and Supplementary file, Table S3). The higher score of modules was regarded as the more meaningful modules in the PPI network. 


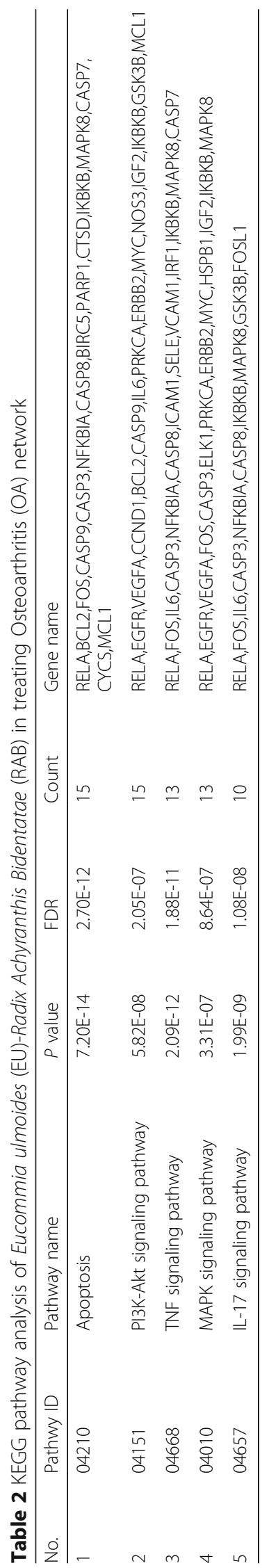




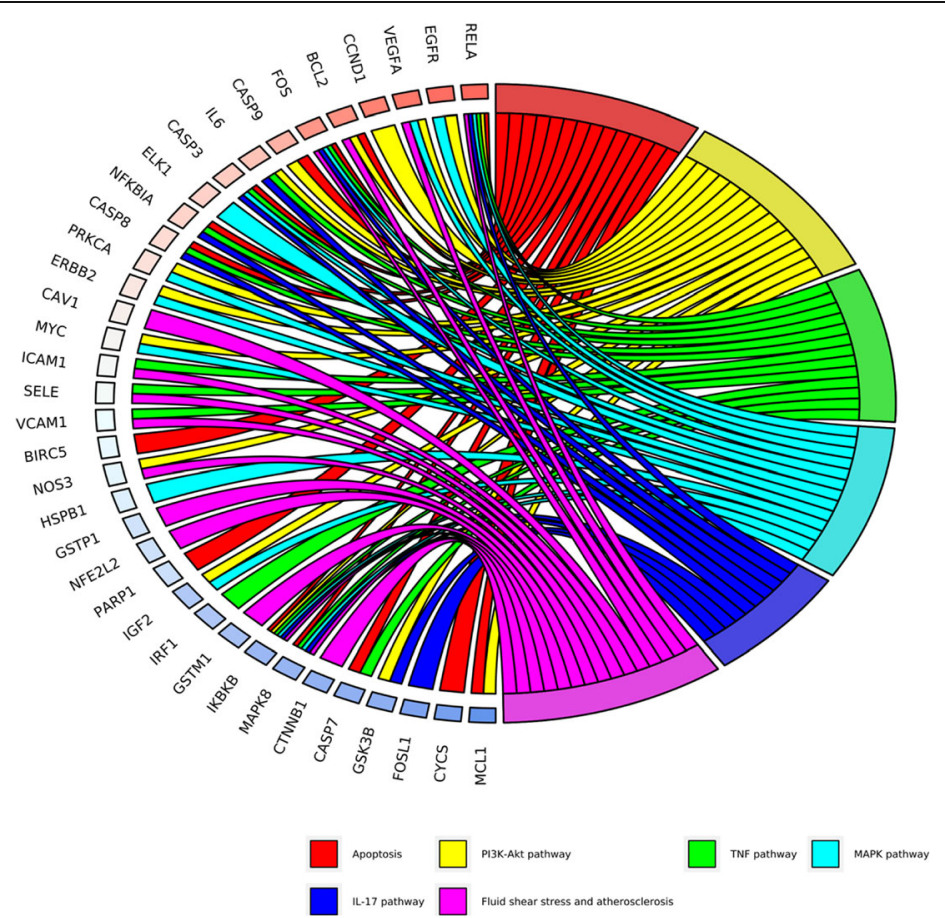

Fig. 3 The KEGG enrichment analysis of genes of Eucommia ulmoides-Radix Achyranthis Bidentatae against Osteoarthritis. Different colors on the right side of the graph represent different signal pathways, and the left side is the core gene with relevance. The more lines in the pathway, the more genes are enriched

\section{Verification of compound-target interaction}

Due to lack of corresponding ligands of a few targets in the PDB, EGFR (PDB id: $1 \mathrm{~m} 17$ ), ESR1 (PDB id: 1r5k), MAPK8 (PDB id: 3pze), GSK3 3 (PDB id: 1q3d) and IKBKB (PDB id: 4kik) were selected based on the core genes, signal pathway and research of osteoarthritis as successfully constructed proteins receptor for molecular docking verification. In molecular docking, if the ligand can bind to one or more amino acid residues with an $\mathrm{H}$ bond, $\mathrm{H}-\pi$ bond or $\pi-\pi$ bond in the active site (also called active pocket) of the receptor and participates in the process of conformation change, energy complementation, etc., then the small molecule ligand can combine with the receptor to form a stable structure [28]. The binding atoms, binding sites, and binding energy intuitively show the interaction and stability of the docking model. In general, the stable docking model has negative binding energy, lower energy score, the stronger binding ability of ligand-receptor, and a more stable structure $[29,30]$. In this study, each protein was successfully docked with 4 bioactive compounds (including quercetin, kaempferol, wogonin, baicalein) derived from the

Table 3 Virtual docking of four bioactive ingredients from Eucommia ulmoides (EU)-Radix Achyranthis Bidentatae (RAB) for Osteoarthritis (OA) targets

\begin{tabular}{|c|c|c|c|c|c|c|c|}
\hline \multirow[t]{2}{*}{ No } & \multirow[t]{2}{*}{ Compound } & \multirow[t]{2}{*}{ Structure } & \multicolumn{5}{|c|}{ Binding Energy $/\left(\mathrm{kcal}^{\prime} \mathrm{mol}^{-1}\right)$} \\
\hline & & & EGFR & ESR1 & MAPK8 & GSK3 $\beta$ & IKBKB \\
\hline 1 & quercetin & & -6.9327 & -6.8972 & -7.1388 & -6.1622 & -7.1583 \\
\hline 2 & kaempferol & & -7.0913 & -6.3109 & -6.6991 & -6.1155 & -6.5508 \\
\hline 3 & wogonin & & -6.4374 & -6.4663 & -6.7080 & -6.1687 & -6.9841 \\
\hline 4 & baicalein & & -6.3727 & -6.5548 & -6.9782 & -5.2388 & -6.3864 \\
\hline
\end{tabular}




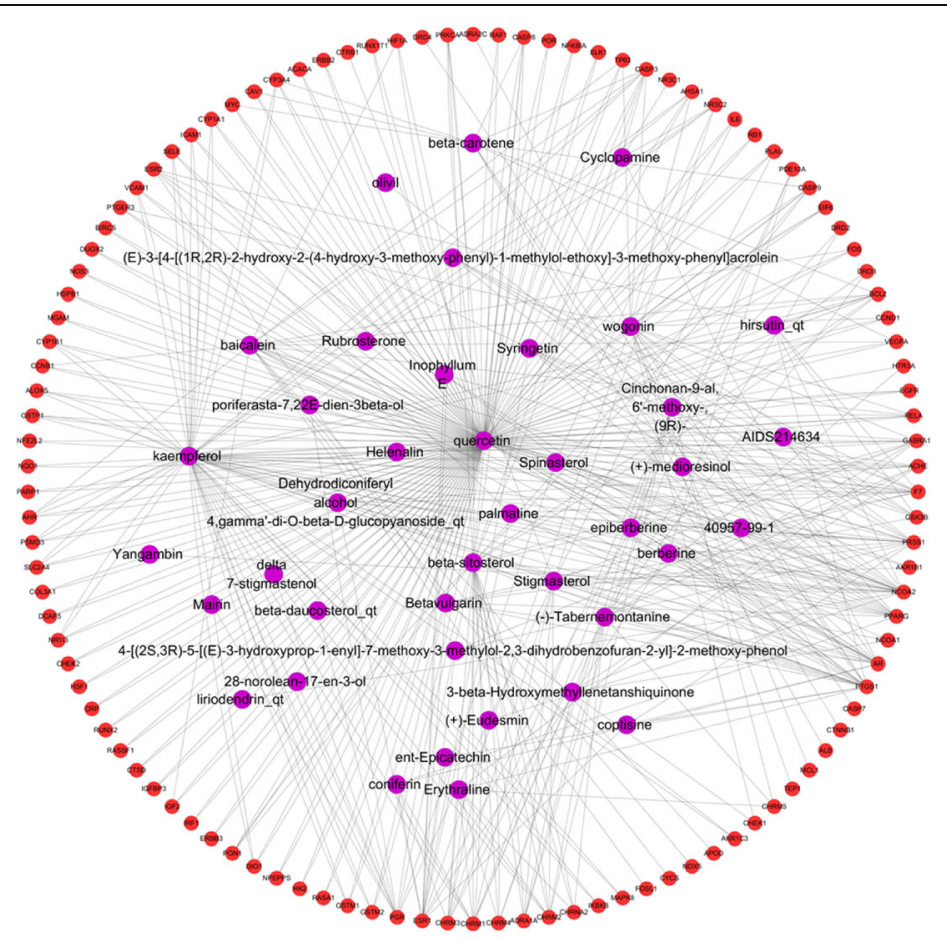

Fig. 4 Potential targets network of Eucommia ulmoides (EU)-Radix Achyranthis Bidentatae (RAB). The potential target network of EU-RAB is constructed using 50 compounds and 110 potential targets. Purple and red cycles represent compound and target respectively. Lines stand for the relation of compounds and target nodes

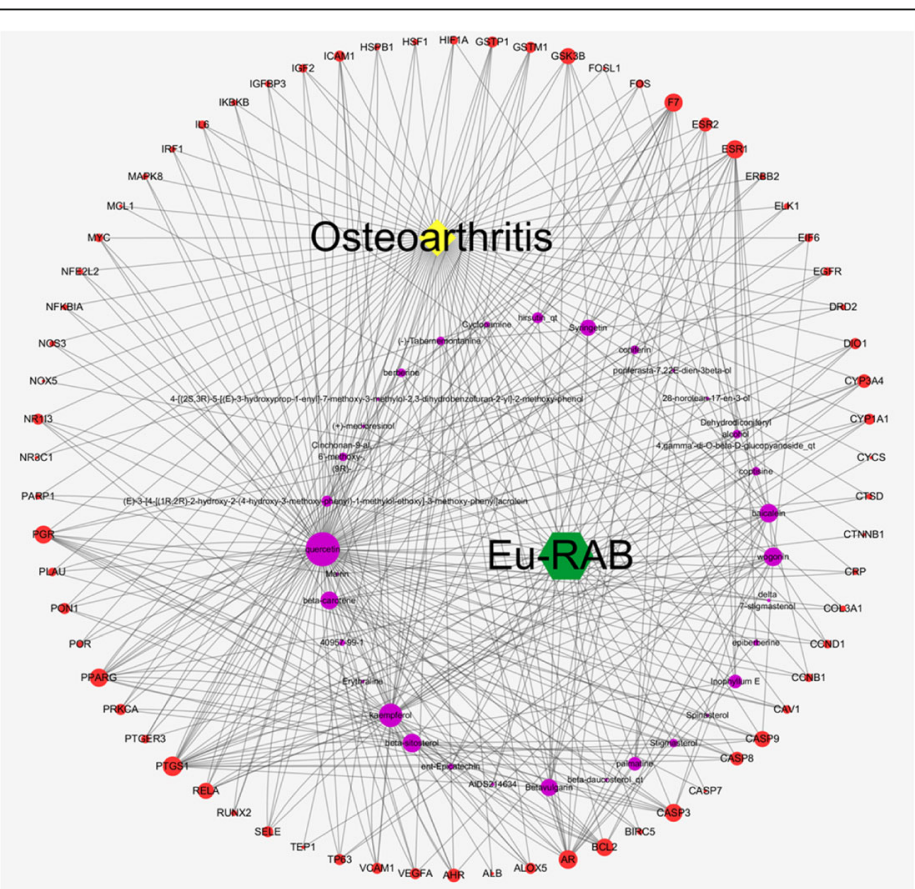

Fig. 5 Compound-target network of Eucommia ulmoides (EU)-Radix Achyranthis Bidentatae (RAB) antiOsteoarthritis (OA). The Compound-target network of EU-RAB anti-OA is constructed using 50 compounds and 68 co-targets, containing 103 nodes and 379 edges. Purple, red, yellow, and green cycles represent compound, target, osteoarthritis, and EU-RAB, respectively. Lines stand for the relation of compounds and target nodes. In all compounds and targets, the lager of node, the higher degree of constituent 
compound-target network, and acquired a stable docking model with a specific binding site, binding distance, and binding atom (Table 3 and Fig. 8). For example, Fig. 8a indicates that quercetin formed an $\mathrm{H}$ bond conjugation with Met 769 (3.23 $\AA$ ) in EGFR. The ESR1-quercetin complex was stabilized in PHE 404 (4.18 $\mathrm{A}, 3.95 \AA$ ) by two $\pi-\mathrm{H}$ bonds (Fig. 8e). Quercetin interacted with MAPK8 via five $\mathrm{H}$ bonds and one $\pi-\mathrm{H}$ bond on $\mathrm{SO} 4$ 402 (3.06 ̊), SO4 402 (3.17 ̊), SO4 402 (3.48 $)$ ), MET 108 (3.00 ̊), GLU 109 (3.15 and VAL 40 (3.87 $\AA$ ) (Fig. 8i). The GSK3 $\beta$-quercetin complex was connected to ASP 133 $(2.97 \AA)$ and ARG 141 (3.08 $\AA$ ) by two $\pi-H$ bonds (Fig. 8m). IKBKB and quercetin were bound in MET 96 (3.25 $)$ ) and GLY 27 (2.76 $)$ ) by two H bonds (Fig. 8q). Therefore, quercetin interacted stably with these targets through an $\mathrm{H}$ bond or $\pi-\mathrm{H}$ bond, and the specific action sites were Met 769, PHE 404, SO4 402, MET 108, GLU 109, VAL 40, ASP 133, ARG 141, MET 96 and GLY 27. Besides, compared with other targets, the docking energy of quercetin with IKBKB $\left(-7.1583 \mathrm{kcal} \cdot \mathrm{mol}^{-1}\right)$ was the lowest, indicating that the binding ability and stability of quercetin-IKBKB complex was higher compared with the other targets.

\section{Discussion}

Network pharmacology is a rational approach used to analyze the potential biological mechanism of TCM in the treatment and prevention of various diseases, and especially in chronic and frequently-occurring disease, such as osteoarthritis [20,31]. Previous studies have confirmed that EU-RAB plays an important role in the treatment of OA $[5,6]$, but the mechanism of action is still not fully understood. Therefore, in the present study, for the first time, network pharmacology was performed to systematically uncover the mechanism of action of EU-RAB against OA. Moreover, the stable molecular docking model of the compound-target exhibited effective binding in representative compounds and hub OA targets, which further verified the inner links between EU$\mathrm{RAB}$ and $\mathrm{OA}$. These typical computer models/figures highlighted the direct effect of EU-RAB on OA, and also showed "multiply compounds, multiply targets, multiply pathways" potential mechanisms.

Through the screening of active ingredients and analysis of compound-target network, quercetin, kaempferol, wogonin, and baicalein were identified to be the most

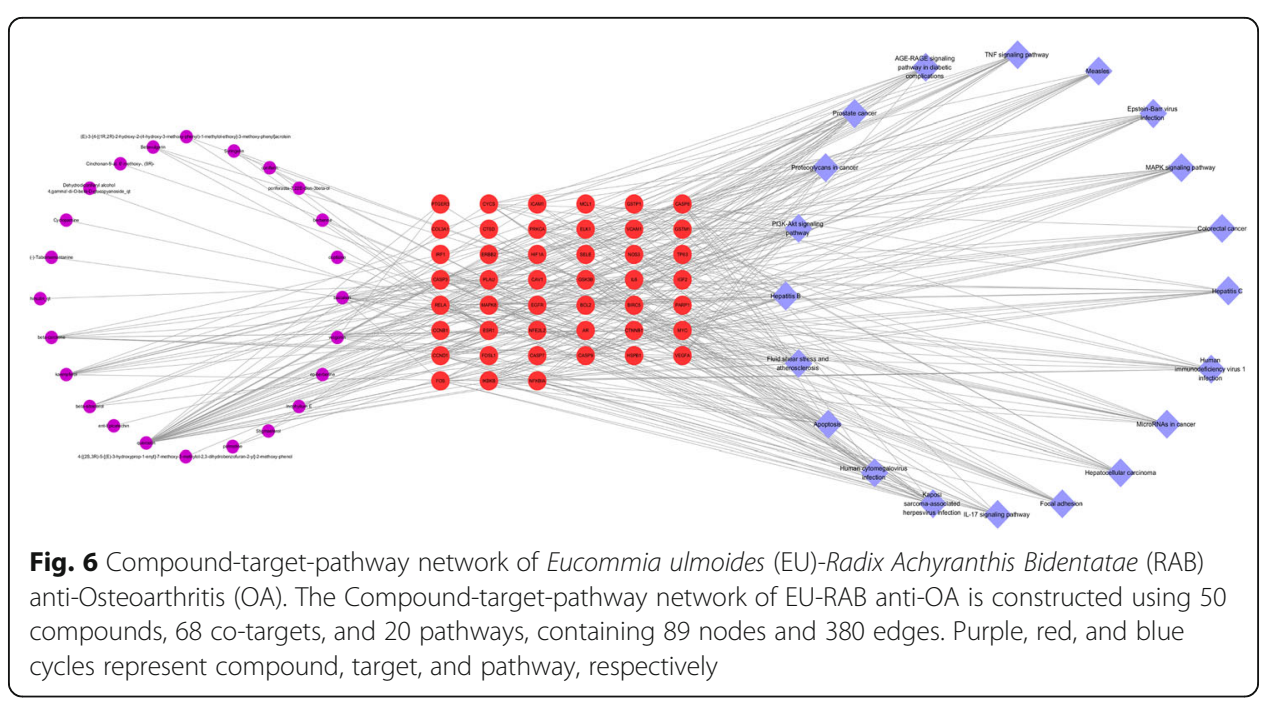




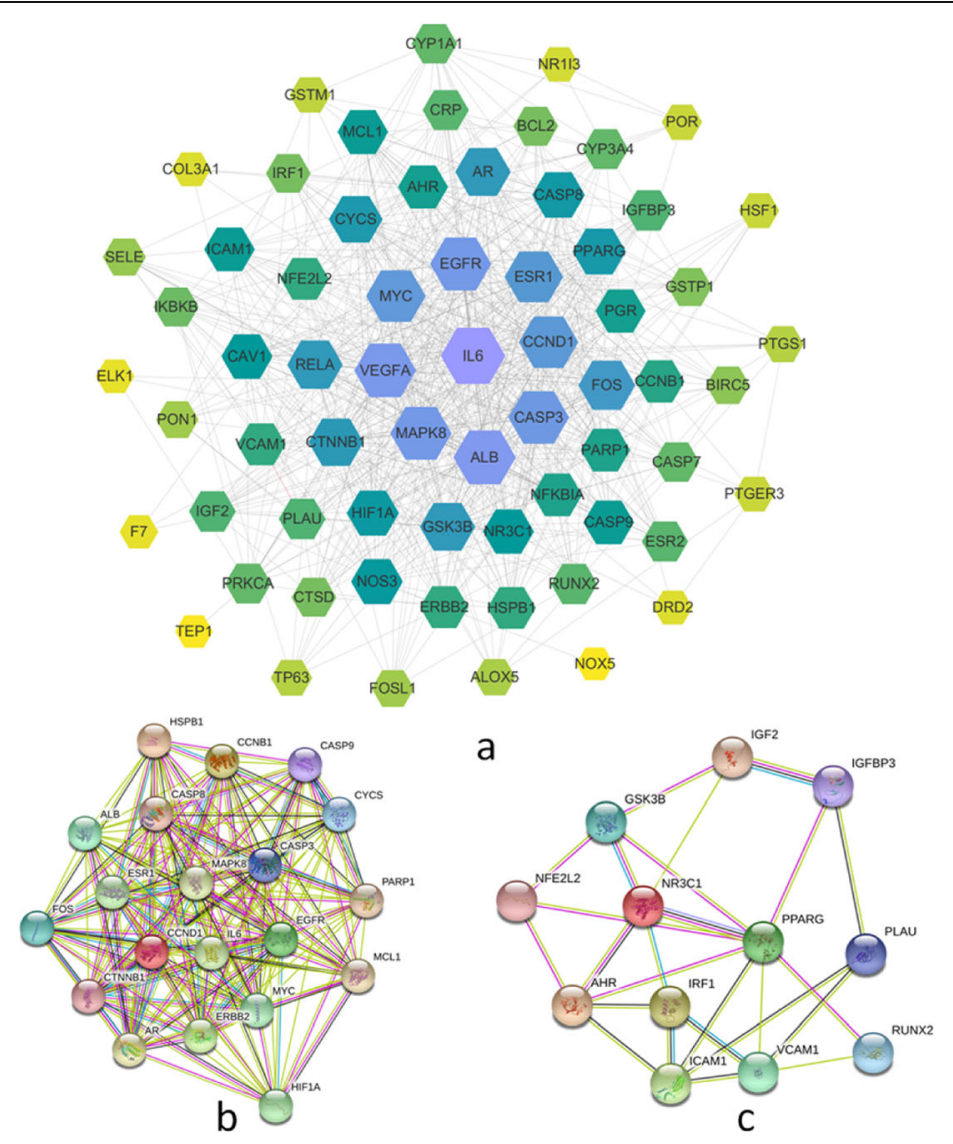

Fig. 7 PPI network of Eucommia ulmoides (EU)-Radix Achyranthis Bidentatae (RAB)-Osteoarthritis (OA) genes. EU-RAB-OA genes PPI network (a) contains 68 nodes and 699 edges. Core genes have a higher degree and are positively correlated with node size and color depth, including, IL-6, VEGFA, EGFR, MAPK8, CASP3, MYC, CCND1, and ESR1. Two clusters are discovered in the PPI network. Cluster 1(b) includes 28 nodes and 290 edges, and Cluster 2(c) includes 12 nodes and 25 edges

important active ingredients of EU-RAB in the treatment of OA. Previous studies have shown that quercetin promotes chondrocytes proliferation and acts on inflammatory injury to accelerate cartilage repair [32]. Increasingly evidence has associated kaempferol with the inflammation process. The anti-inflammation function of kaempferol is manifested in the modulation of cytokines and suppression of MAPK pathways [33]. Wogonin and baicalein are major flavones constituents with chondroprotective effects. Wogonin possesses a tight binding affinity with chondrocytes genomic DNA and stabilizes the DNA fragmentation to protect chondrocytes [34]. The anti-apoptosis and anticatabolic effects of baicalein related to OA are embodied in an increase of glycosaminoglycan and type II collagen [35]. Notably, these active ingredients of EU-RAB synergistically exert anti-inflammation, anti-apoptosis, and maintenance of cartilage homeostasis effects, which can be regarded as potential therapeutic strategies against OA.

By integrating all the targets of EU-RAB and OA, 68 targets related to OA were identified. The 68 targets were used to construct PPI for use in highlighting pivotal targets and interaction degree with others, such as IL6, EGFR, VEGFA, CSAP3, ESR1, GSK3 $\beta$, etc. In the entire target network of EU-RAB against OA, these 

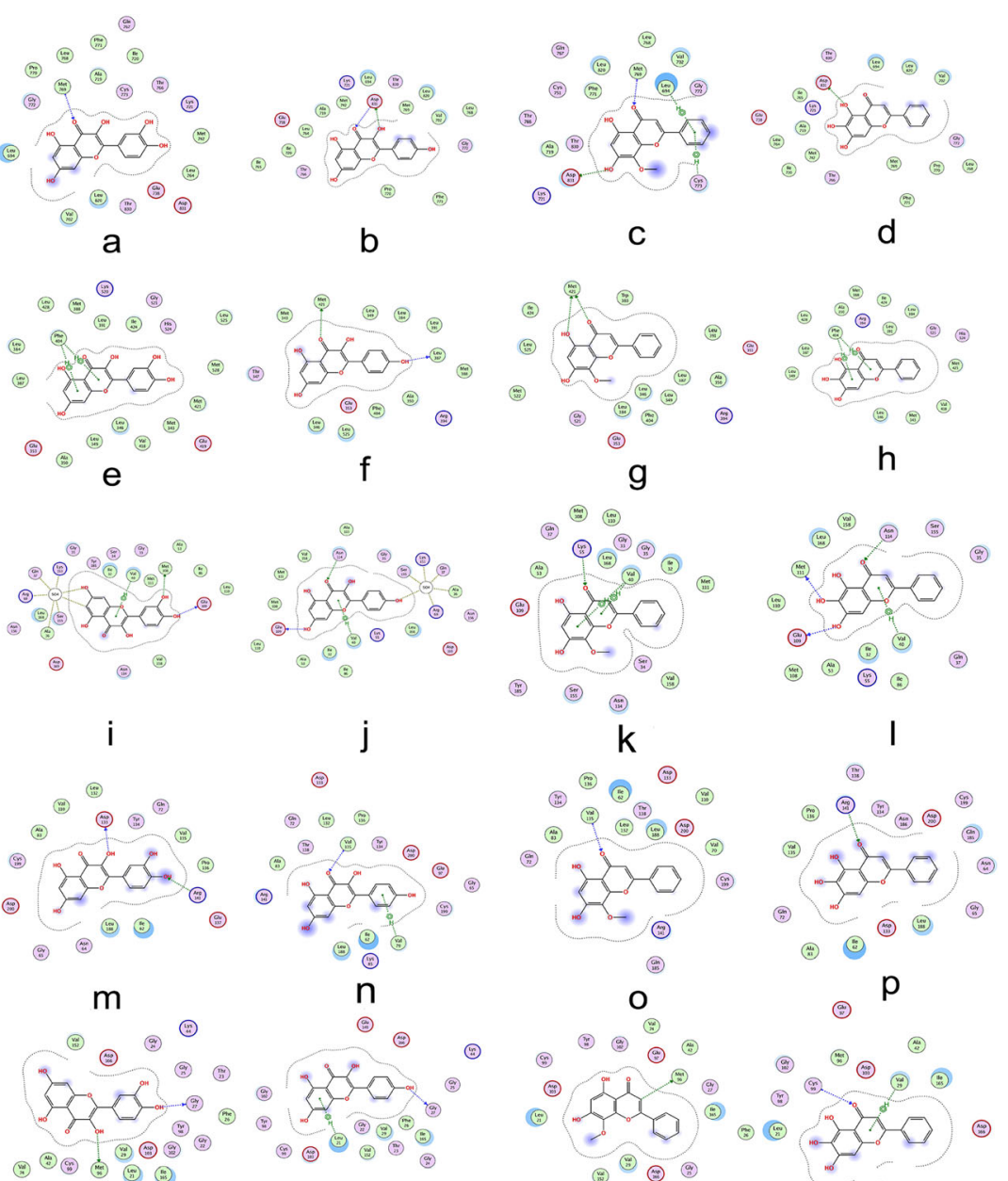

(:)

q

$r$

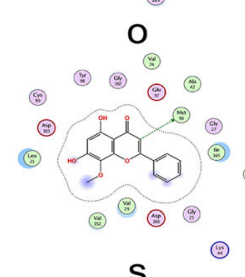

$p$

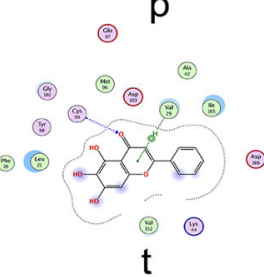

polar $\ldots . .+$ sidechain acceptor

acidic *... sidechain donor

Obasic $\rightarrow \rightarrow$ backbone acceptor

greasy +... backbone donor proximity contour

ligand

exposure

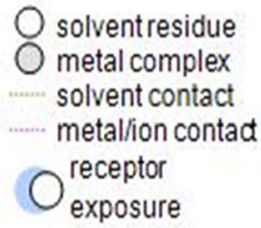

(ㅇ) arene-arene

(0) $\mathrm{H}$ arene-H

(0)+ arene-cation

Fig. 8 Virtual docking of bioactive ingredients from Eucommia ulmoides-Radix Achyranthis Bidentatae for Osteoarthritis targets. The molecular docking of quercetin, kaempferol, wogonin, and baicalein with EGFR $(\mathbf{a}, \mathbf{b}, \mathbf{c}, \mathbf{d}), \operatorname{ESR} 1(\mathbf{e}, \mathbf{f}, \mathbf{g}, \mathbf{h}), \operatorname{MAPK} 8(\mathbf{l}, \mathbf{j}, \mathbf{k}, \mathbf{l}), \operatorname{GSK3} \beta(\mathbf{m}, \mathbf{n}, \mathbf{o}, \mathbf{p})$, and IKBKB $(\mathbf{q}, \mathbf{r}, \mathbf{s}, \mathbf{t})$ are shown

targets showed a rich interaction with others and were more likely to produce a cascade effect for exerting antagonistic effects on OA. Moreover, in the C-T-P network, gene targets acting on multiple pathways at the same time were also found, indicating the importance of specific targets in the entire OA bioinformatics network. For instance, MAPK8 (degree $=14$ ) appeared in apoptosis, TNF, and MAPK signaling pathways, and both PI3/Akt and IL-17 signaling pathways contained GSK3 $\beta$ (degree $=16$ ). This also reflected the "multiply targets-multiply pathways" regulation mechanism of EU-RAB. IL-6 is a key pro-inflammatory mediator cytokine related to bone resorption and degradation of the extracellular matrix [36]. VEGFR serves as an important target participating in the mediation of 
inflammation, cell proliferation, and pain management [37, 38]. Caspase3 and GSK3 $\beta$ as the key apoptotic cytokines medicating DNA fragmentation and oxidant stress injury resulting in the acceleration of chondrocyte apoptosis [39, 40]. Therefore, the above targets were believed to be involved in the key molecular action site of EU-RAB anti-OA.

GO and KEGG analysis revealed that "apoptosis", "TNF signal", "IL-17 signal", "PI3K/Akt signal pathway" and "MAPK signal pathway" were related to the mechanism of EU-RAB in the treatment of OA. In this study, "apoptosis" mapped the most key targets and possessed the highest degree of enrichment, and was the most dominant signaling way of EU-RAB against OA. Combined with hub targets of $\mathrm{BP}$ and MF analysis, the anti-apoptotic effect of EU-RAB reflected on the mediation of oxidative stress (GO:0006979, GO:0034599) and apoptotic signaling (GO: 2001233, GO:0097153). Besides, the active ingredients of EU-RAB were found to possess a good anti-apoptosis effect via inhibition of inflammatory induction or directly suppressing cellular stress state [32-35]. Simultaneously, molecular docking results showed that a representative apoptotic cytokine, GSK3 $\beta$ had a good binding affinity with the above active ingredients, which also confirmed the direct antiapoptotic effect of EU-RAB. Aberrant apoptosis resulted in a decrease of chondrocytes number and abnormal morphology and function, which led to difficulties in sustaining the mechanical stress balance of the extracellular matrix as well as induce cartilage matrix degradation [41, 42]. Furthermore, accompanying disorders of physicochemical factors or inflammatory cytokines during aberrant apoptosis produced a high concentration of reactive oxygen species leading to oxidative stress which disturbed the tranquility of cartilage homeostasis $[43,44]$. Inflammatory cascade, apoptotic protein, and NF-K $\beta$ signaling are the main promoters of Tumor Necrosis Factor (TNF) driving the multiply biological function, especially the inflammatory and apoptotic response [45]. In this study, EU-RAB was found to inhibit the activity of TNF signaling by affecting 15 targets such as IL6, IKBKB, Caspase3, etc. Besides, as an important member of the NF-K $\beta$ family, IKBKB formed a good visualization model with the main active ingredients in molecular docking analysis. These results showed the good target effect of EU-RAB on TNF signaling. Interleukin-17 (IL-17) signaling played a key role in the pathophysiological process of Immunomodulation and infection, osteoarthritis susceptibility [46], and pain control [47]. MAPK is a member of the serine-threonine protein kinases family and a transduction center for cell multidirectional changes involved in the development of OA [48]. PI3K/AKT signaling, is an important signal transduction site associated with the pathophysiological process of intracellular metabolism, including cell proliferation, autophagy, and apoptosis [49].

In this study, network pharmacology was used to elaborate on the potential antiosteoarthritis effect of EU-RAB and this effect was visualized by molecular docking for verification. This scientific and systematic theoretical study provides a basis for future pharmacological studies to further explore the mechanism of EU-RAB in the treatment of OA.

\section{Conclusion}

Traditional Chinese medicine has a long history for thousands of years in the treatment of osteoarthritis. As a typical representative, EU-RAB has been reported to play a protective effect through multiple targets and pathways in OA. For the first time, this study systematically expounds on the mechanism of action of EU-RAB anti-OA using network 
pharmacology. Besides, this effect is visualized through molecular docking to verify this relationship. In this study, 50 main bioactive compounds of EU-RAB anti-OA are identified. Moreover, IL-6, EGFR, VEGFA, ESR1, CASP3, GSK3 $\beta$, MAPK8, etc., are the pivotal genes enriched to signaling transduction pathways (e.g apoptosis, TNF signaling) and for EU-RAB to exert its anti-apoptosis and anti-inflammatory effect on OA. Therefore, the analysis of molecular biological networks based on multiply target-pathway interaction using network pharmacology not only contributes to deepening the understanding of EU$\mathrm{RAB}$ against $\mathrm{OA}$ but also provides a pharmacology basis for further research.

\section{Supplementary information}

Supplementary information accompanies this paper at https://doi.org/10.1186/s13040-020-00221-y.

Additional file 1: Table S1. Active compounds of Eucommia ulmoides (EU)-Radix Achyranthis Bidentatae (RAB). Table S2. Potential targets of Eucommia ulmoides (EU)-Radix Achyranthis Bidentatae (RAB). Table S3. Cluster network of target genes of Eucommia ulmoides (EU)-Radix Achyranthis Bidentatae (RAB) against Osteoarthritis (OA)

\section{Abbreviations}

OA: Osteoarthritis; TCM: Traditional Chinese Medicine; EU: Eucommia ulmoides; RAB: Radix Achyranthis Bidentatae; TCMSP: Traditional Chinese Medicine Systems Pharmacology; BATMAN-TCM: Bioinformatics Analysis Tool for Molecular mechanism of Traditional Chinese Medicine; OB: Oral Bioavailability; DL: Druglikeness; ADME: Absorption, Distribution, Metabolism and Excretion; GO: Gene ontology; KEGG: Kyoto Encyclopedia of Genes and Genomes; PPI: Protein-protein interaction; BP: Biological Processes; CC: Cell Components; MF: Molecular Functions; Caspase3: Cysteinyl aspartatespeciwc proteinase 3; GSK3ß: Glycogen Synthase Kinase 3 beta; IKBKB: Inhibitor of nuclear factor kappa-B kinase subunit beta; ESR1: Estrogen receptor; EGFR: Epidermal growth factor receptor; MAPK8: mitogen-activated protein kinase; VEGF: Vascular endothelial growth factor; IL-6: Interleukin-6; TNF: Tumor Necrosis Factor; IL-17: Interleukin-17

\section{Acknowledgements}

Not applicable.

\section{Authors' contributions}

JGH and XH conceived and designed the study. SBZ collected the data. JGH and ZWJ performed the data analysis, $\mathrm{JGH}$ and $\mathrm{XH}$ wrote the manuscript. All authors are responsible for reviewing data. All authors read and approved the final manuscript.

\section{Funding}

This work is financially supported by the Hunan Provincial Natural Science Foundation of China (No. 2019JJ40224).

\section{Availability of data and materials}

The data used to support the findings of this study are available from the corresponding author upon request, unless there are legal or ethical reasons for not doing so.

Ethics approval and consent to participate

Not applicable.

\section{Consent for publication}

Not applicable.

\section{Competing interests}

The authors declare that there are no conflicts of interest in relation to this work.

Received: 27 May 2020 Accepted: 9 August 2020

Published online: 28 August 2020

\section{References}

1. Hunter DJ, S B-Z. Osteoarthritis. Lancet (London, England). 2019:393(10182):1745-59.

2. Vina ER, Kwoh CK. Epidemiology of osteoarthritis: literature update. Curr Opin Rheumatol. 2017;30(2):1.

3. Mandl LA. Osteoarthritis year in review 2018: clinical. Osteoarthr Cartil. 2019;27(3):359-64.

4. Li L, Haiqing L, Weimei S, et al. Insights into the action mechanisms of traditional Chinese medicine in osteoarthritis. Evid Based Complement Alternat Med. 2017;2017:1-13.

5. He X, Wang X, Fang J, et al. The genus Achyranthes : a review on traditional uses, phytochemistry, and pharmacological activities. J Ethnopharmacol. 2017;203:260-78.

6. He X, Wang JH, Li MX, et al. Eucommia ulmoides Oliv.: Ethnopharmacology, phytochemistry and pharmacology of an important traditional Chinese medicine. J Ethnopharmacol. 2014;151(1):78-92.

7. Chen F-P, Chang C-M, Hwang S-J, et al. Chinese herbal prescriptions for osteoarthritis in Taiwan: analysis of national health insurance dataset. Bmc Complementary \& Alternative Medicine. 2014;14(1):91. 
8. LiPing YANG, Cheng SP, Zhang W, et al. Bioinformatics analysis of different Core drugs on intervention of osteoarthritis based on literature. J Tradit Chin Med. 2016;57(23):2042-6.

9. Lei ZHANG, JuBao LI, Yan ZENG, et al. Literature study on the application of orally taken Chinese medicinal for knee osteoarthritis. J Tradit Chin Med. 2013;54(7):608-10.

10. Xie G-p, Jiang N, Wang S-n, et al. Eucommia ulmoides Oliv. Bark aqueous extract inhibits osteoarthritis in a rat model of osteoarthritis. J Ethnopharmacol. 2015;162:148-54.

11. Zhang R, Pan Y-L, Hu S-J, et al. Effects of total lignans from Eucommia ulmoides barks prevent bone loss in vivo and in vitro. J Ethnopharmacol. 2014;155(1):104-12.

12. Xu XX, Zhang X-H, Diao Y, et al. Achyranthes bidentate saponins protect rat articular chondrocytes against interleukin$1 \beta$-induced inflammation and apoptosis in?Vitro. Kaohsiung J Med Sci. 2017;33(2):62-8.

13. Zhang $X, X u X, X u T$, et al. $\beta$-Ecdysterone suppresses interleukin-1 $\beta$-induced apoptosis and inflammation in rat chondrocytes via inhibition of NF-KB signaling pathway. Drug Dev Res. 2014;75(3):195-201.

14. Weng $X$, Lin $P$, Liu F, et al. Achyranthes bidentata polysaccharides activate the Wnt/ $\beta$-catenin signaling pathway to promote chondrocyte proliferation. Int J Mol Med. 2014;34(4):1045-50.

15. Lee Won-Yung, Lee Choong-Yeol, Kim Youn-Sub, et al. The Methodological Trends of Traditional Herbal Medicine Employing Network Pharmacology. Biomolecules. 2019; 9:362.

16. Zhang Z, Yi P, Huang J, et al. Integrated network pharmacology analysis and serum metabolomics to reveal the cognitive improvement effect of Bushen Tiansui formula on Alzheimer's disease. J Ethnopharmacol. 2019;1:112371.

17. Li S, ZHANG B. Traditional Chinese medicine network pharmacology: theory, methodology and application. Chin J Nat Med. 2013;11(2):110-20

18. Ru J, Li P, Wang J, et al. TCMSP: a database of systems pharmacology for drug discovery from herbal medicines. Journal of Cheminformatics. 2014;6(1):13.

19. Liu Z, Guo F, Wang Y, et al. BATMAN-TCM: a bioinformatics analysis tool for molecular mechANism of traditional Chinese medicine. Sci Rep. 2016;6:21146.

20. Chao H, Zheng C, Yan L, et al. Systems pharmacology in drug discovery and therapeutic insight for herbal medicines. Brief Bioinformatics. 2014;15:710-33.

21. Huang J, Cheung F, Tan HY, et al. Identification of the active compounds and significant pathways of yinchenhao decoction based on network pharmacology. Mol Med Rep. 2017;16(4):4583-92.

22. Xu X, Zhang W, Huang C, et al. A novel chemometric method for the prediction of human oral bioavailability. Int J Mol Sci. 2012;13:6964-82.

23. You JS, Li CY, Chen W, et al. A network pharmacology-based study on Alzheimer disease prevention and treatment of Qiong Yu Gao. BioData Min. 2020;13:2.

24. Ma C, Wang L, Xie X. GPU accelerated chemical similarity calculation for compound library comparison. J Chem Inf Model. 2011;51:1521-7.

25. Rebhan M, Chalifa-Caspi V, Prilusky J, et al. GeneCards: a novel functional genomics compendium with automated data mining and query reformulation support. Bioinformatics. 1998;14(8):656-64.

26. Ada H, Scott AF, Amberger JS, et al. Online Mendelian inheritance in man (OMIM), a knowledgebase of human genes and genetic disorders. Nucleic Acids Res. 2002;30(1):52-5.

27. Altaf-Ul-Amin M, Shinbo Y, Mihara K, et al. Development and implementation of an algorithm for detection of protein complexes in large interaction networks. BMC Bioinformatics. 2006;7(1):207.

28. Yan Wei, Li Shawn Xiang, Wei Minjie, et al. Identification of MMP9 as a novel key gene in mantle cell lymphoma based on bioinformatic analysis and design of cyclic peptides as MMP9 inhibitors based on molecular docking. Oncol. Rep. 2018:40: 2515-2524.

29. Rahman Noor, Muhammad ljaz, Gul-E-Nayab, et al. Molecular Docking of Isolated Alkaloids for Possible a-Glucosidase Inhibition. Biomolecules. 2019;9(10): 544.

30. Li L, Qiu H, Liu M, Cai Y. A network pharmacology-based study of the molecular mechanisms of Shaoyao-Gancao decoction in treating Parkinson's disease. Interdiscip Sci. 2020;12(2):131-44.

31. Tang Huaqi, He Shuaibing, Zhang Xinyue, et al. A Network Pharmacology Approach to Uncover the Pharmacological Mechanism of XuanHuSuo Powder on Osteoarthritis. Evid Based Complement Alternat Med. 2016;2016:3246946.

32. Hu Y, Gui Z, Zhou Y, et al. Quercetin alleviates rat osteoarthritis by inhibiting inflammation and apoptosis of chondrocytes, modulating synovial macrophages polarization to M2 macrophages. Free Radic Biol Med. 2019;145:146-60.

33. Huang $X$, Pan $Q$, Mao Z, et al. Kaempferol inhibits interleukin-1 $\beta$ stimulated matrix metalloproteinases by suppressing the MAPK-associated ERK and P38 signaling pathways. Mol Med Rep. 2018;18(3):2697-04.

34. Khan NM, Ahmad I, Ansari MY, et al. Wogonin, a natural flavonoid, intercalates with genomic DNA and exhibits protective effects in IL-1 $\beta$ stimulated osteoarthritis chondrocytes. Chem Biol Interact. 2017;274:13-23.

35. Zhang $X$, Zhu Y, Chen $X$, et al. Baicalein ameliorates inflammatory-related apoptotic and catabolic phenotypes in human chondrocytes. Int Immunopharmacol. 2014;21(2):301-8.

36. Wojdasiewicz P, Poniatowski ŁA, Szukiewicz D. The role of inflammatory and anti-inflammatory cytokines in the pathogenesis of osteoarthritis. Mediat Inflamm. 2014;2014:1-19.

37. Shotaro T, Kentaro U, Gen I, et al. Vascular endothelial growth factor expression and their action in the synovial membranes of patients with painful knee osteoarthritis. BMC Musculoskelet Disord. 2018;19(1):204.

38. Chen H, Yun T. MiR-15a-5p regulates viability and matrix degradation of human osteoarthritis chondrocytes via targeting VEGFA. Bioscience Trends. 2016;10(6):482-8.

39. Xi-Bin K, Qun C, Gao Y, et al. SP600125 blocks the proteolysis of cytoskeletal proteins in apoptosis induced by gas signaling molecule (NO) via decreasing the activation of caspase-3 in rabbit chondrocytes. Eur J Pharmacol. 2018;824:40-7.

40. Serena G, Manuela M, Daniela P, et al. Lithium chloride dependent glycogen synthase kinase 3 inactivation links oxidative DNA damage, hypertrophy and senescence in human articular chondrocytes and reproduces chondrocyte phenotype of obese osteoarthritis patients. PLoS One. 2015;10(11):e0143865.

41. Edith C, Biserka R, Céline D, et al. Insights on molecular mechanisms of chondrocytes death in osteoarthritis. Int J Mol Sci. 2017;17(12):2146. 
42. Pountos I, Giannoudis PV. Modulation of cartilage's response to injury: can chondrocyte apoptosis be reversed? Injury. 2017:48(12):2657-69.

43. Blanco FJ, Rego I, Ruiz-Romero C. The role of mitochondria in osteoarthritis. Nat Rev Rheumatol. 2011;7(3):161-9.

44. Liu H, Li Z, Cao Y, et al. Effect of chondrocyte mitochondrial dysfunction on cartilage degeneration: a possible pathway for osteoarthritis pathology at the subcellular level. Mol Med Rep. 2019;20(4):3308-16.

45. $E V Q, D D S, G ~ V L, S E, K G$ A protein-protein interaction map of the TNF-induced NF-KB signal transduction pathway Scientific data 2018;5:180289.

46. Bai Y, Gao S, Liu Y, et al. Correlation between Interleukin-17 gene polymorphism and osteoarthritis susceptibility in Han Chinese population. BMC Med Genet. 2019;20(1):20.

47. Wei M. Correlation of IL-17 level in synovia and severity of knee osteoarthritis. Medical Science Monitor International Medical Journal of Experimental \& Clinical Research. 2015;21:1732-6.

48. Zhang Y, Pizzute T, Pei M. A review of crosstalk between MAPK and Wnt signals and its impact on cartilage regeneration. Cell \& Tissue Research. 2014;358(3):633-49.

49. Xue J-F, Zhong-Min S, Jian Z, et al. Inhibition of PI3K/AKT/mTOR signaling pathway promotes autophagy of articular chondrocytes and attenuates inflammatory response in rats with osteoarthritis. Biomed Pharmacother. 2017:89:1252-61.

\section{Publisher's Note}

Springer Nature remains neutral with regard to jurisdictional claims in published maps and institutional affiliations.

\section{Ready to submit your research? Choose BMC and benefit from:}

- fast, convenient online submission

- thorough peer review by experienced researchers in your field

- rapid publication on acceptance

- support for research data, including large and complex data types

- gold Open Access which fosters wider collaboration and increased citations

- maximum visibility for your research: over $100 \mathrm{M}$ website views per year

At $\mathrm{BMC}$, research is always in progress. 